Musées, Patrimoine et Culture scientifiques et techniques

182 | 2019

mars-avril 2019

\title{
Construire une exposition scientifique participative. Le projet Questions de Sciences, Enjeux Citoyens
}

Vera de Sousa et Claire Garraud

\section{(2) OpenEdition \\ 12 Journals}

Édition électronique

URL : http://journals.openedition.org/ocim/2310

DOI : $10.4000 /$ ocim.2310

ISSN : 2108-646X

Éditeur

OCIM

\section{Édition imprimée}

Date de publication : 1 mars 2019

Pagination : 18-25

ISSN : 0994-1908

\section{Référence électronique}

Vera de Sousa et Claire Garraud, « Construire une exposition scientifique participative. Le

projet Questions de Sciences, Enjeux Citoyens », La Lettre de I'OCIM [En ligne], 182 | 2019, mis en ligne le 01 mars 2020, consulté le 17 mars 2020. URL : http://journals.openedition.org/ocim/2310 ; DOI :

https://doi.org/10.4000/ocim.2310

Ce document a été généré automatiquement le 17 mars 2020.

Tous droits réservés 
Construire une exposition scientifique participative. Le projet Questions de Sciences, Enjeux Citoyens

Vera de Sousa et Claire Garraud 
Dans l'exposition Trajectoire, l'expo qui interroge nos mobilités, ce module muséographique illustre les résultats contre-intuitifs des mouvements de foule.

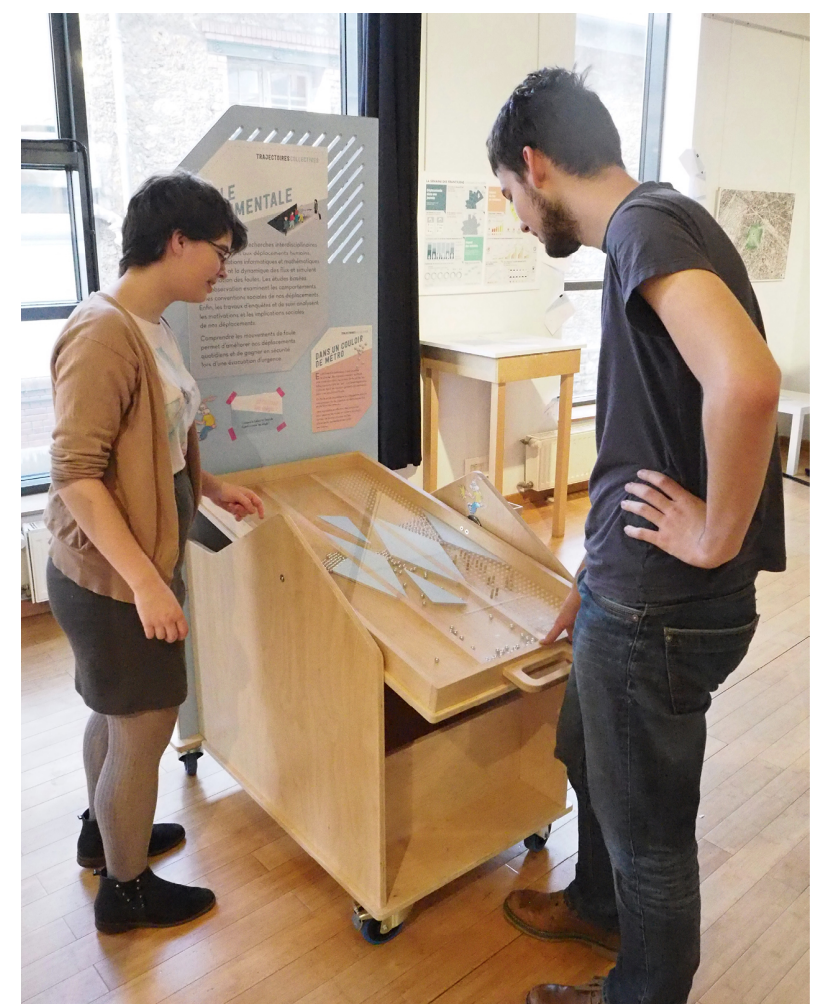

(c) ESPGG

1 Ayant pour ambition de développer le dialogue entre habitants, chercheurs et élus, le projet Questions de Sciences, Enjeux Citoyens (QSEC) existe depuis 2009. Il a depuis connu plusieurs évolutions en terme de format, de publics ou encore d'échelle de participation. Mis en œuvre sur la région île-de-France par huit associations de médiation scientifique réunies en consortium ${ }^{1}$, le dispositif invite des habitants de la région francilienne constitués en "groupes citoyens " à explorer une thématique scientifique à fort impact sociétal à travers des parcours de réflexion : rencontres avec des spécialistes scientifiques, débats en groupe et visites culturelles. Les participants se saisissent ainsi des enjeux sociétaux liés aux questions scientifiques et techniques d'actualité.

2 À partir de 2014, le projet QSEC évolue et devient QSEC ${ }^{2}$. Le financement initial accordé par la région île-de-France se complète d'une subvention du programme d'Investissements d'Avenir (PIA). Le dispositif intègre alors deux éléments complémentaires aux parcours de réflexions initiaux : une exposition itinérante modulable co-construite avec des citoyens et la mise en œuvre d'un plan d'action local afin d'ancrer le dispositif sur le territoire. Articulé autour de ces trois piliers fondamentaux, le dispositif se déploie sur toute l'île-de-France et, dans chaque département, le lieu d'accueil de l'exposition devient le point d'ancrage du projet pour faire rayonner les thématiques abordées par cet outil concret et accessible qu'est l'exposition itinérante. Après une première exposition, Air, l'expo qui inspire ${ }^{2}$, le projet QSEC $^{2}$ explore une nouvelle thématique depuis novembre 2017 avec Trajectoires, l'expo qui interroge nos mobilités. L'occasion pour nous de revenir sur la mise en œuvre du dispositif et l'opportunité de diffusion de culture scientifique qu'il crée. 
Comme de nombreux dispositifs de culture scientifique, le projet QSEC $^{2}$ s'est doté dès sa création d'un conseil scientifique, le « Comité d'Orientation Scientifique et Pédagogique "(COSP). Cette instance composée de spécialistes de diverses disciplines assure l'encadrement scientifique du projet et se compose de deux entités :

un comité scientifique permanent ${ }^{3}$ qui accompagne la mise en œuvre du projet dans la durée et un comité scientifique thématique rassemblant des experts en lien direct avec la thématique de l'exposition en cours. Le COSP valide les champs scientifiques traités dans l'exposition et en garantit la vocation sociale.

Affiche de l'exposition Trajectoires, l'expo qui interroge nos mobilités

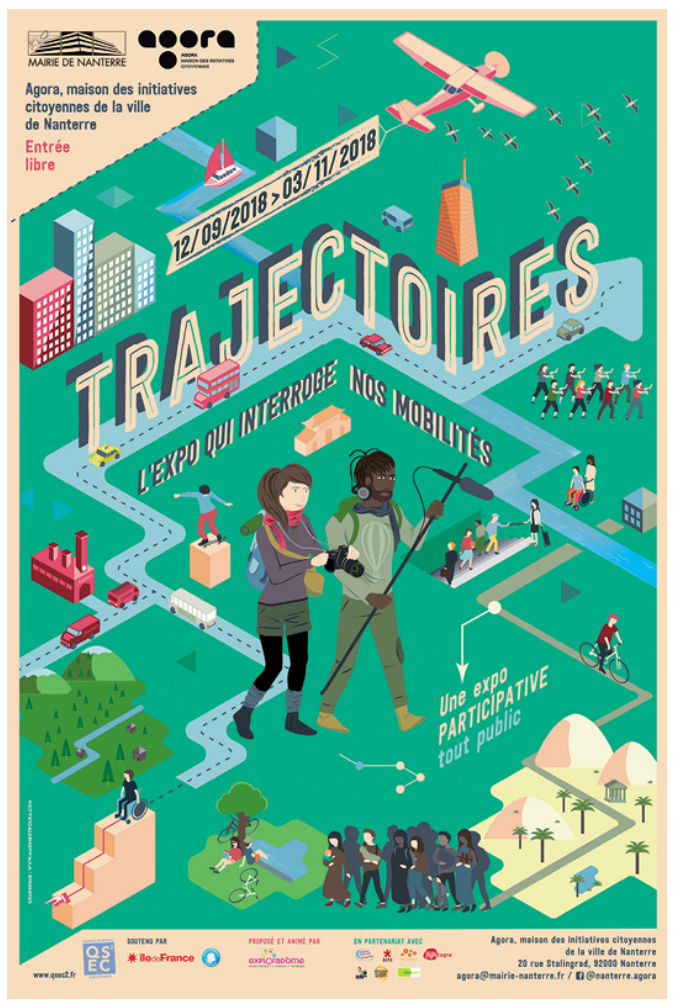

(c) Graphisme : www.marineetpolka.com

\section{Les groupes d'habitants au cœur du dispositif}

Depuis sa création, le projet $\mathrm{QSEC}^{2}$ intègre la participation des habitants franciliens dans sa mise en œuvre et les valeurs qu'il porte sont inhérentes à cette participation : inclusion, "mise en capacité " des participants (empowerment) et développement de l'esprit critique. En faisant appel à des habitants volontaires dans chaque département pour constituer des groupes citoyens, les associations partenaires respectent trois paramètres essentiels : diversité des profils, mixité sociale et culturelle et respect de la parité.

6 Ces huit groupes, un par département, sont accompagnés par chaque association partenaire dans un parcours de réflexion qui se compose d'une dizaine de rendez-vous. Ces derniers proposent aux participants des rencontres avec des scientifiques et d'autres acteurs en lien avec la thématique, des visites culturelles et des temps 
d'échanges entre participants, animés par des professionnels de la médiation scientifique.

7 Ces parcours viennent alimenter la conception de l'exposition à différentes échelles. Lors de la première exposition, Air, l'expo qui inspire, la participation des habitants s'est effectuée sur deux niveaux : trois groupes étaient impliqués dans la définition des thèmes traités dans les supports de l'exposition tandis que les autres groupes exploraient une sous-thématique précise et proposaient une " production » à intégrer dans l'itinérance de l'exposition (vidéos, textes, objets). "Cela permet de concrétiser des informations dans des cas réels, la science est plus accessible»; "Cela donne un ton plus proche de celui des publics, rend l'exposition plus vivante, apporte un autre regard que celui des scientifiques " (témoignages visiteurs de l'exposition, 2017). L'exposition, qui a mobilisé près de 200 partenaires locaux, a reçu plus de 50000 visiteurs sur neuf lieux d'accueil répartis sur les huit départements de la Région Île-de-France.

Pour la nouvelle exposition Trajectoires, l'expo qui interroge nos mobilités, les huit groupes de participants conçoivent chacun un support de médiation intégré dans le mobilier muséal. Le parcours de réflexion s'articule entièrement autour de cette "production ». Partant d'abord d'une exploration très générale de la thématique, les mobilités, le groupe citoyen choisit ensuite un angle plus précis qu'il s'approprie et parcourt en le reliant avec sa propre expertise et ses connaissances. Cette appropriation ajoute une perspective sociale et culturelle au dispositif proposé ensuite aux visiteurs de l'exposition. Pour que chaque individu puisse prendre part aux échanges, les médiateurs scientifiques mettent en place une méthodologie qui sort le participant d'une posture de spectateur ou de récepteur passif. Cette approche rappelle que tout le monde peut être un détenteur ou un producteur de savoirs à un moment ou un autre, et que ces savoirs sont valorisables.

9 "Cette séance était consacrée à la conception de la contribution citoyenne pour l'exposition. En début de séance nous sommes revenus sur les différentes idées que nous avions et nous avons débattu sur les supports et les éléments à intégrer. (...) La majorité a souhaité "donner de la voix" aux mots qui représentent la mobilité humaine " (participant du groupe citoyen des Hautsde-Seine qui a produit le module des mots de la migration intégré dans l'exposition, mars 2017). 
Design de l'exposition Trajectoires, l'expo qui interroge nos mobilités

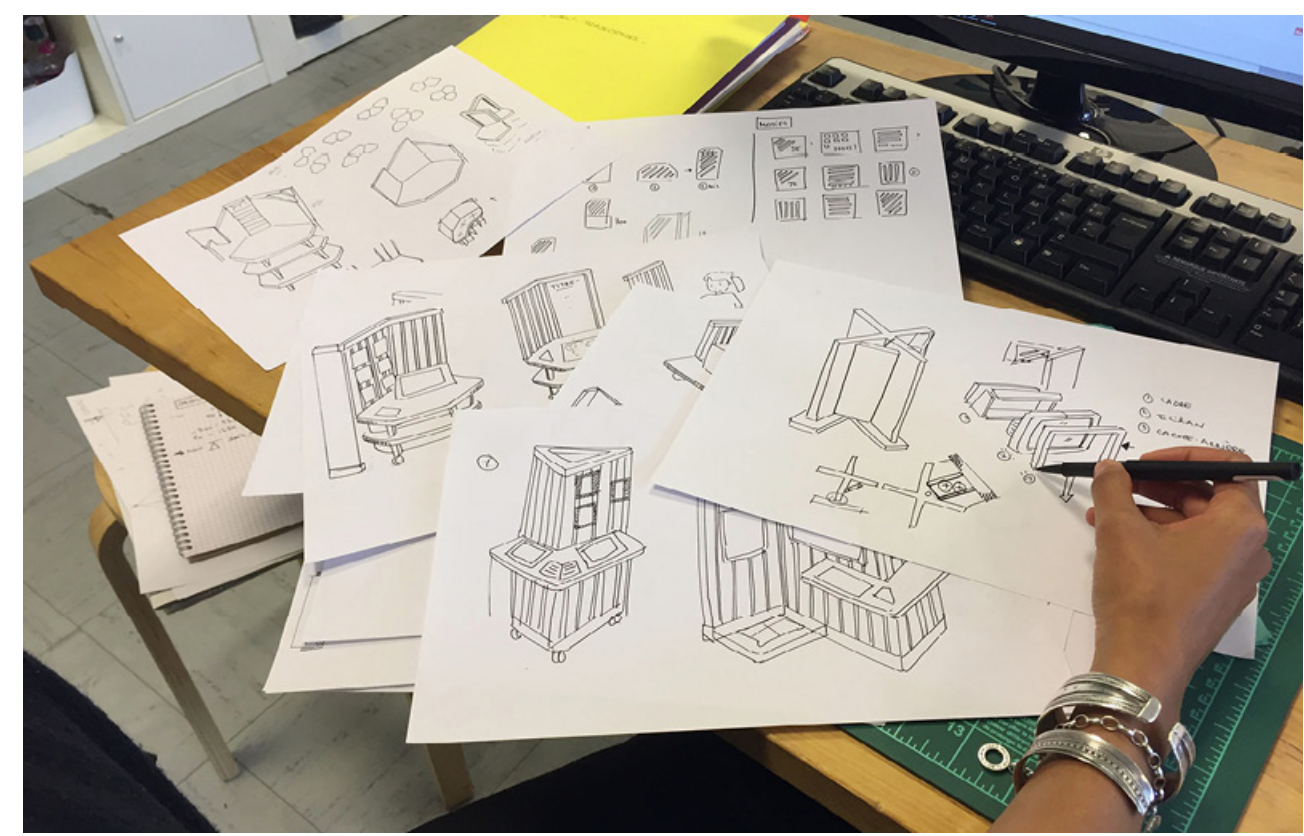

(C) Exploradôme/Apis

Devenant alors outil de mise en capacité et d'expression pour les participants des groupes citoyens, le parcours de réflexion confirme cette volonté de répondre aux différents enjeux de la culture scientifique, technique et industrielle que porte le projet $\mathrm{QSEC}^{2}$ : favoriser la capacité critique des citoyens dans les questionnements « Sciences/ Société ", valoriser les processus de discussion qui mènent aux décisions publiques et porter la vision d'une responsabilité sociétale de la recherche scientifique.

11 Ce dispositif d'engagement avec les groupes citoyens se décline ensuite tout au long de l'exposition et chaque niveau de participation trouve sa place dans le projet. Que cela se matérialise par la mise en place d'un projet inspiré par la présence de l'exposition, par une visite de groupe et des échanges ou par une proposition formulée sur des espaces d'expression, la participation des visiteurs est sans cesse encouragée et accompagnée. Qu'elle s'exprime sous la forme d'un engagement long ou ponctuel, elle concrétise la démarche globale du projet $\mathrm{QSEC}^{2}$. 
Module de l'exposition conçu par le groupe citoyen des Hauts-de-Seine : témoignages autour des mots de la migration.

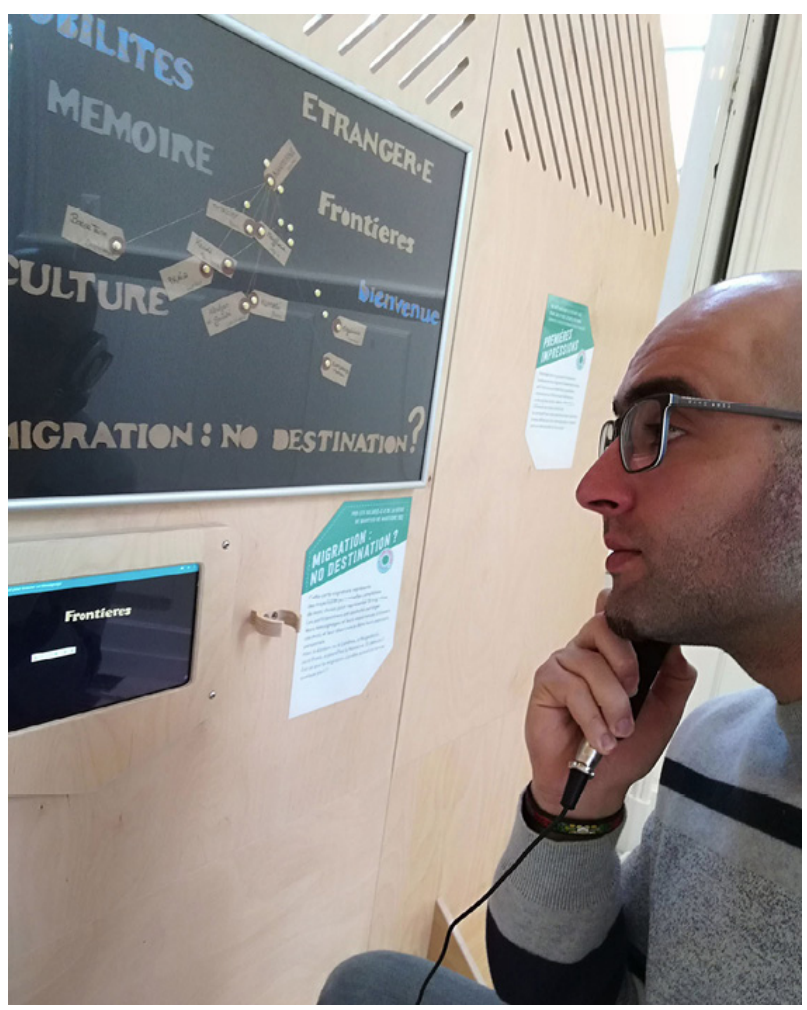

(c) Science Ouverte

Dispositif sur les migrations, actuelles et historiques, de l'espèce humaine

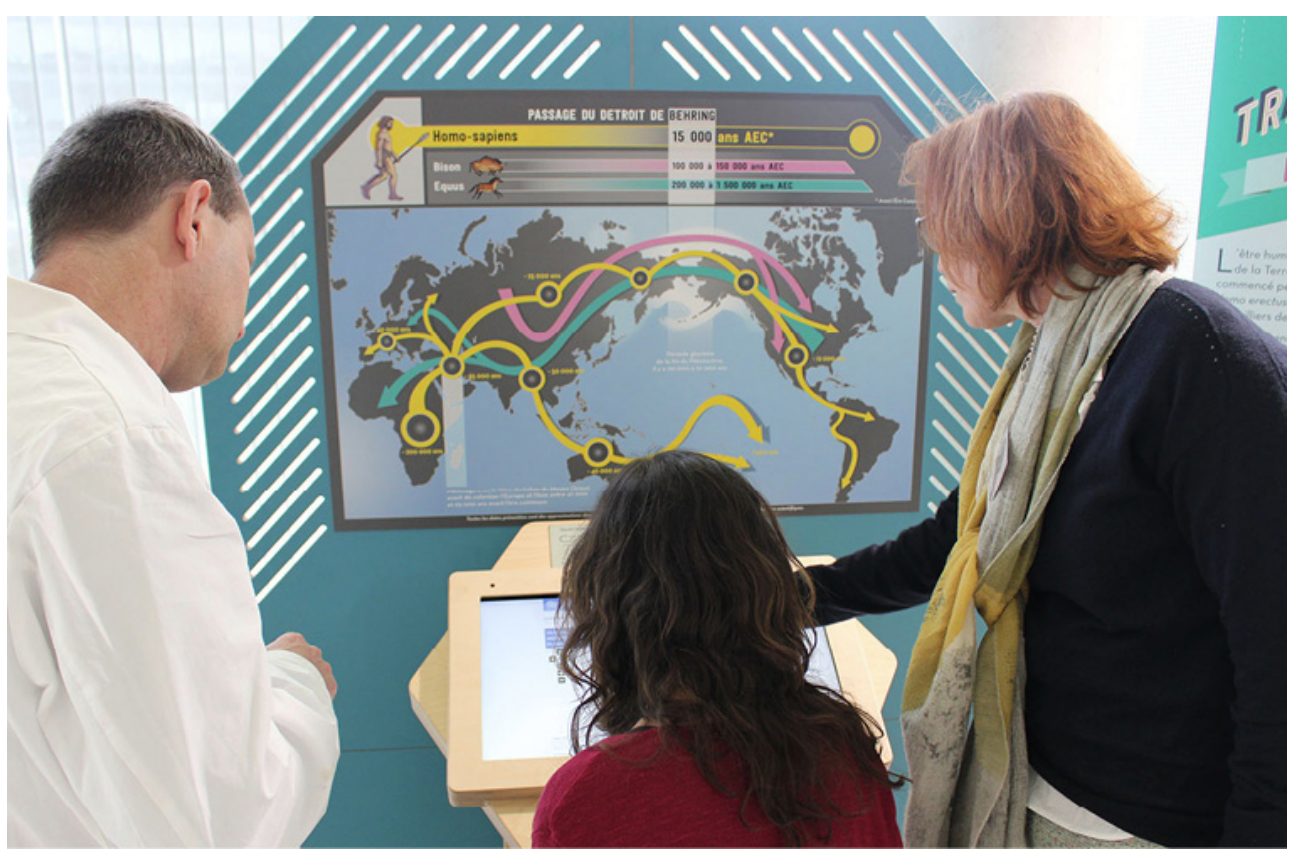

(c) Exploradôme 


\section{L'exposition, concrétisation d'un espace relationnel des sciences en société}

Une exposition est avant tout un espace, un lieu matériel et défini présentant des outils d'exploration pour les visiteurs. Son schéma de conception est souvent linéaire : recherche du thème et conception muséographique avec l'appui d'un comité d'experts. La conception matérielle et la fabrication sont ensuite confiées à des designers, des scénographes ou autres corps de métiers participant à la fabrication.

Dans Air, l'expo qui inspire, les questions relatives au réchauffement climatique, à la pollution atmosphérique ou la qualité de l'air en ville y trouvent tout naturellement leur place. Les visiteurs peuvent naviguer d'une thématique à l'autre, d'un champ scientifique à un autre tout en s'interrogeant sur les impacts et les enjeux sociétaux présentés. Cette intersection entre notions scientifiques et préoccupations sociétales est un pilier du projet. Les contributions des groupes citoyens intégrées dans l'exposition permettent de croiser les regards entre sciences et société et d'illustrer ce dialogue.

Dans Trajectoires, l'expo qui interroge nos mobilités, ce croisement est rendu encore plus visible auprès des visiteurs grâces aux supports co-conçus par les groupes citoyens et complètement intégrés dans le parcours de visite. L'exposition aborde la mobilité comme la somme de deux concepts, le mouvement (du corps, des individus, des populations) et la société (histoire, économie, urbanisme, culture). Elle se structure en trois pôles, qui explorent les interactions entre mouvement et société à différentes échelles : celle de l'individu, celle du groupe et de la ville, et celle de la planète. Dans le pôle " trajectoires individuelles », ce sont les problématiques autour du mouvement du corps qui sont explorées : posture et démarche, handicap et mobilité réduite, mobilité virtuelle. Les questions relatives aux mobilités urbaines (transports, urbanisme) et plus généralement aux mobilités collectives (mouvements de foule, bouchons...) sont traitées dans le pôle " trajectoires collectives ». Enfin, le pôle " trajectoires mondiales " ouvre la réflexion sur des échelles de temps et d'espace élargies : migrations de l'espèce humaine au fil des âges, mouvements migratoires actuels, migrations culturelles (monnaie, langue).

Le mobilier muséal : le défi de l'itinérance, de la participation et de la modularité

L'exposition Trajectoires intègre un mobilier muséal qu'il est possible d'installer sur une surface minimum de $100 \mathrm{~m} 2$.

Conçue et fabriquée par APIS (Atelier de Prototypage pour l'Investigation Scientifique), elle répond à quatre objectifs fondamentaux :

- être solide pour résister aux nombreuses itinérances ;

- être modulable pour s'adapter à des lieux aux configurations variées ;

- être interactive avec des manips et des dispositifs numériques invitant les visiteurs à tester et expérimenter ; 
- valoriser les contributions citoyennes.

Le pôle « trajectoires mondiales » lors de l'itinérance à l'Espace des sciences Pierre-Gilles de Gennes

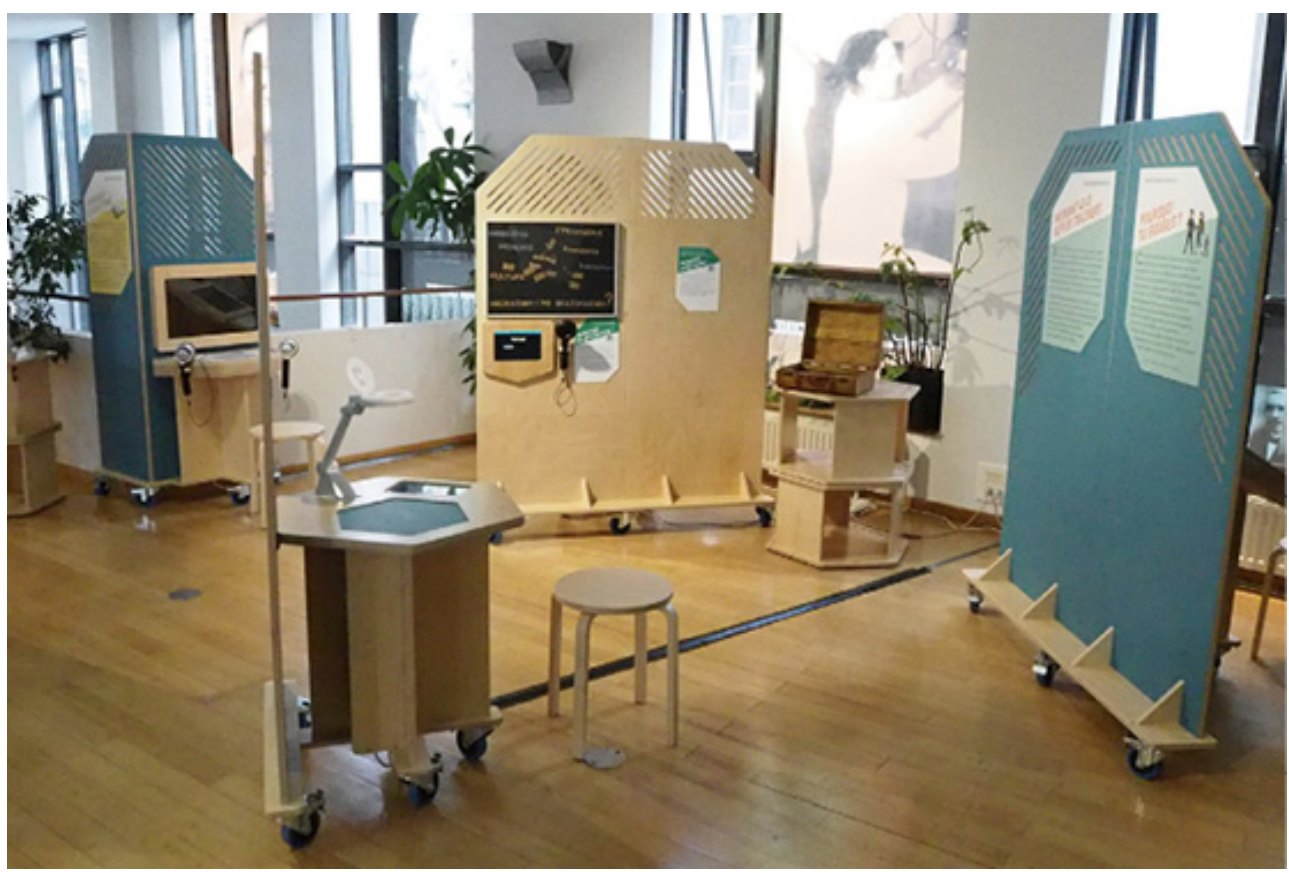

(c) ESPGG

15 Le groupe citoyen accompagné par l'association Traces (Paris) a choisi, comme terrain de réflexion, l'échangeur de Bagnolet, ce grand complexe routier à l'est de Paris. Sa mise en œuvre a soulevé de nombreuses interrogations et réflexions au sein du territoire. Les participants du groupe ont décidé de raconter son évolution et ses conséquences sur la vie quotidienne des habitants. Leurs productions offrent une vision créative et engagée pour aborder les impacts de cette complexe infrastructure.

L'exploration de la migration par quatre groupes citoyens (Essonne, Hauts-de-Seine, Seine-Saint-Denis et Val-de-Marne) est aussi l'occasion de partager avec les publics des expériences plus personnelles à travers des témoignages audio, des textes ou des cartes. Ces productions intégrées dans le pôle «trajectoires mondiales » en font aussi un lieu de partage de témoignages.

Avec ces contributions empathiques et vivantes, l'exposition devient un matériel perméable et crée un environnement relationnel à la thématique du projet. Elle rend visible tous les liens entre sciences et sociétés, entre enjeux scientifiques, enjeux politiques et enjeux citoyens. L'espace qu'elle met à disposition des publics invite les visiteurs à recevoir cette exposition de manière active, participative et ludique. L'exposition, conçue pour intégrer un lieu d'accueil différent à chaque itinérance, se veut également évolutive et intègre un mobilier spécifique pour accueillir de nouvelles contributions des publics. Éléments éphémères ou durables, ces nouveaux objets viennent compléter l'exposition d'un regard directement en lien avec le territoire dans lequel elle s'intègre.

18 Lors de son itinérance à Paris, au sein de l'Espace des Sciences Pierre-Gilles de Gennes (voir encadré ci-dessus), Trajectoires s'est vue complétée de différents éléments comme 
par exemple, une vidéo présentant des visuels de recherches scientifiques en cours de l'École Supérieure de Chimie et de Physique Industrielles de la ville de Paris (ESPCI) ou encore une exposition prêtée par le lycée Vauquelin présentant les portraits de ses élèves nouvellement arrivés sur le territoire français (dispositif Classe de Scolarisation et d'Insertion - CSI). Pendant sa présentation à la médiathèque du Chatelet-en-Brie en Seine-et-Marne, des élèves du collège Rosa Parks ont rédigé des histoires migratoires sous l'impulsion de leurs enseignants. Ces textes collectés sont maintenant proposés à la lecture des visiteurs de l'exposition.

L'itinérance de l'exposition à travers la région Île-de-France est imaginée comme un rendez-vous capable d'opérer une mise en relation entre publics, scientifiques et décideurs politiques. Sa simple présence ne suffit pas toujours et c'est pourquoi elle s'accompagne d'un plan d'action local indispensable à la diffusion du projet mobilisant de nombreux acteurs locaux.

L'exposition Air, l'expo qui inspire à Garges-lès-Gonesses en juillet 2016

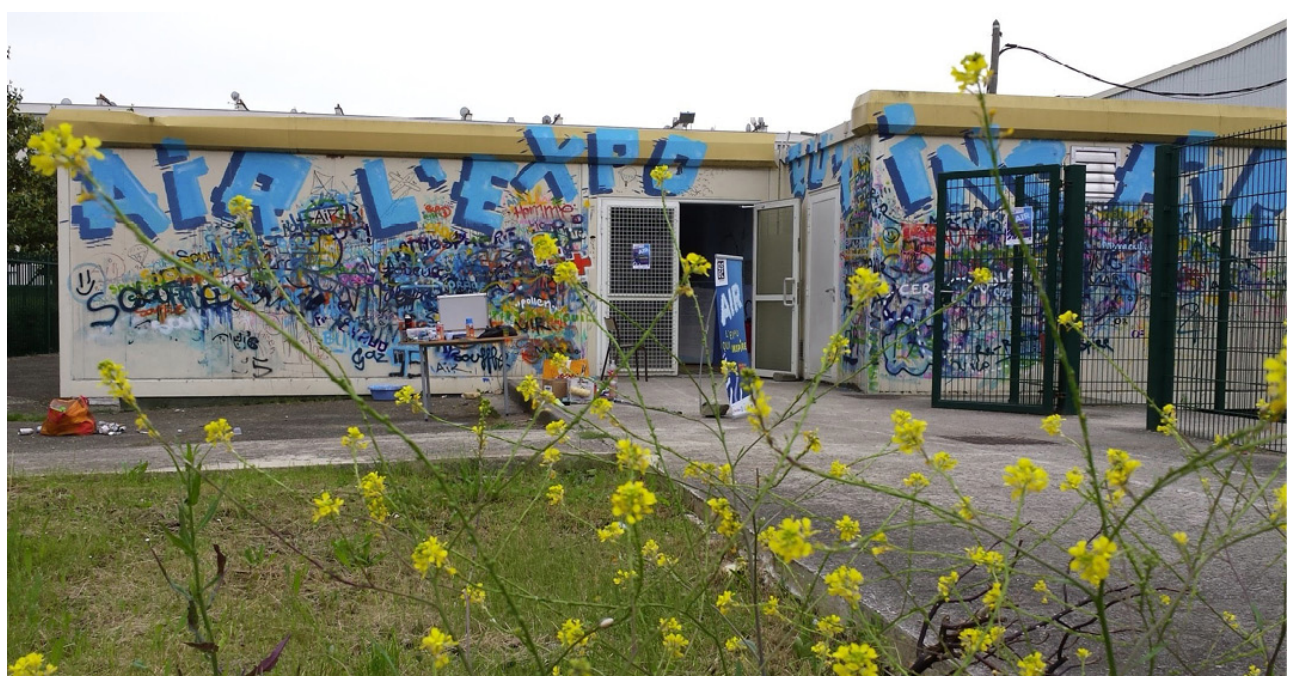

(c) Les Petits Débrouillards

\section{Engager les publics par un ancrage territorial}

La présence de l'exposition dans un lieu est un événement culturel qui crée des dynamiques relationnelles entre usagers du lieu, habitants du quartier, de la ville, des communes limitrophes et du département tout entier.

21 Le plan d'action local a pour objectif d'accompagner la présence de l'exposition en mobilisant des acteurs locaux (associations, établissements scolaires, services municipaux, lieux culturels, centres socio-culturels). Les associations partenaires travaillent en collaboration avec ces structures et élaborent avec elles une programmation qui place la thématique de l'exposition dans l'agenda culturel du territoire.

Trois temps sont nécessaires pour la mise en place du plan d'action local. Le premier temps correspond à la préparation de l'arrivée de l'exposition sur le territoire et à la mise en relation des partenaires locaux, le deuxième temps à la présence de 
l'exposition et le dernier temps aux effets de la présence du dispositif QSEC ${ }^{2}$ auprès des partenaires et des publics.

Le lieu d'accueil de l'exposition sert de point de départ à l'animation du plan d'action local. Trois des associations partenaires possèdent leur propre lieu : l'Espace des Sciences Pierre Gilles de Gennes pour l'association Traces à Paris, le château de Ladoucette pour Science Ouverte à Drancy et l'Exploradôme pour l'association Savoir Apprendre à Vitry-sur-Seine. Les autres nouent des partenariats avec des structures locales en capacité d'accueillir le dispositif.

Air, l'expo qui inspire a été présentée dans trois médiathèques (médiathèques des Cités Unies à Savigny-le-Temple, Jean Cocteau à Massy et Albert Camus à Évry), une île de loisirs à Saint-Quentin-en-Yvelines, à l'Agora, maison des initiatives citoyennes de Nanterre (92) et dans un préfabriqué municipal à Garges-lès-Gonesse. La typologie des publics que l'espace d'accueil partenaire touche "naturellement » (publics familiaux, publics scolaires, enfants, adolescents ou adultes, publics dits « éloignés » socialement) et les missions sociales portées par le lieu (cohésion sociale, diffusion des sciences, démocratisation culturelle) façonnent complètement le plan d'action local.

"L'exposition a vraiment trouvé sa place dans toutes les activités de la ville pendant deux mois. Tout le monde a joué le jeu! On a décoré la médiathèque avec les productions des enfants confectionnées en amont dans l'optique de la venue de l'exposition. Et le dernier jour, nous avons organisé un événement avec toutes les associations de la commune, en mobilisant des partenaires » (référente du projet en Seine-et-Marne, de l'association Terre Avenir).

Pendant deux mois environ, chaque lieu se fait l'écho du projet et de l'exposition. Mais ce n'est pas tout. L'exposition étant l'espace matériel du dispositif $\mathrm{QSEC}^{2}$, elle n'en limite pas l'action à cet espace. Un maillage d'actions à travers le territoire permet de diffuser plus largement la thématique de l'exposition à travers des actions " hors les murs ». Que ce soit sous la forme d'un ciné-débat, d'un jeu de piste urbain, d'une balade à vélo ou encore d'une visite de laboratoire, ces animations proposées en partenariat avec les acteurs locaux offrent aux publics un cadre large d'accès aux thématiques de l'exposition. La convergence entre ces actions locales et le projet QSEC $^{2}$ fait naitre des variétés de regards et d'échanges qui nourrissent la richesse participative du dispositif.

Quelle influence ou quelle portée l'exposition et son traitement participatif ont pu générer sur le territoire ? D'autres projets ou initiatives citoyennes ont-ils été portés par la suite ? Il paraît difficile de quantifier ce type de retours d'expériences une fois l'exposition partie pour d'autres territoires, toutefois sa présence est un terrain de rencontres fertiles pour des partages de connaissances et de questionnements.

Les partenaires du projet se placent dans une amélioration continue de leur pratique de la médiation scientifique et abordent la fin de l'itinérance de l'exposition comme le commencement d'autres projets que $\mathrm{QSEC}^{2}$ aura permis d'essaimer.

\section{Une évolution dynamique du projet}

Proposer une exposition scientifique dans laquelle le processus de participation des habitants est visible et valorisé donne corps à la dimension démocratique dans le dialogue sciences et société. En proposant, pendant l'itinérance de l'exposition, des temps de rencontres et d'échanges formels ou informels auprès de tous les publics, le projet $\mathrm{QSEC}^{2}$ participe à cette opportunité d'engagement des habitants dans les enjeux 
scientifiques et à cette convergence territoriale nécessaire à la diffusion des savoirs et des connaissances.

Cette convergence se traduit également dans les processus internes au projet. Son coportage par huit associations partenaires et son maillage sur le territoire constituent un véritable challenge pour les actions de CSTI en Île-de-France. Les évolutions du dispositif, ses améliorations continues et sa mise en œuvre plurielle, ont permis de créer un espace d'expérimentation et de professionnalisation pour chacun des acteurs associatifs. Diversifier ses publics, intégrer une démarche participative dans ses animations ou encore mettre en place des partenariats avec des acteurs culturels locaux sont autant d'éléments qui ont enrichi les pratiques de médiation scientifique. Cette dynamique de groupe et ce fonctionnement en réseau favorisant le travail collaboratif ont permis de structurer le partage des compétences et des expériences entre les différents acteurs, de mettre en place de nouveaux processus évolutifs des dispositifs de médiation et d'ouvrir la voie vers de nouveaux projets collaboratifs entre les professionnels de la culture scientifique francilienne.

L'itinérance de Trajectoires, l'expo qui interroge nos mobilités jusqu'en février 2020 est l'occasion de voir à l'œuvre ce processus dynamique d'amélioration continue.

\section{NOTES}

1. Association Traces/Espace des Sciences Pierre-Gilles de Gennes (Paris), Terre Avenir (Seine-etMarne), Association Science Technologie Société (Yvelines), Planète Sciences île-de-France (Essonne), Paris Montagne (Hauts-de-Seine), Science ouverte (Seine-Saint-Denis), Savoir Apprendre/Exploradôme (Val-de-Marne), Les Petits Débrouillards île-de-France (Val-d'Oise).

2. Plus de 51600 visiteurs sur toute l'île-de-France, 9 lieux d'accueil dans les huit départements, plus de 200 partenaires locaux impliqués, $98 \%$ de satisfaction (parmi les 505 répondants aux questionnaires d'évaluation).

3. Julien Bobroff (enseignant-chercheur, laboratoire de Physique des Solides CNRS/université Paris Sud), Jean-Gabriel Ganascia (professeur à l'université Pierre et Marie Curie, Équipe ACASA, laboratoire LIP6), Pierre-Henri Gouyon (professeur au MNHN, à AgroParisTech, à Sciences Po, à l'ENS Paris ; chercheur au sein du laboratoire à l'Institut de Systématique, Évolution et Biodiversité, UMR 7205 MNHN-CNRS-UPMC), Jack Guichard (président de Logistique Associative et Bureau d'étude Littoral, ancien directeur du Palais de la découverte, professeur des universités ENS Cachan), Claudine Hermann (présidente d'honneur de l'association Femmes \& Sciences, professeure honoraire à l'École Polytechnique), Pierre-Benoît Joly (directeur de Recherche à l'Inra, directeur du Laboratoire interdisciplinaire Sciences Innovations Sociétés), Valérie Lallemand-Breitenbach (directrice de recherche à l'Inserm et à l'Institut universitaire d'Hématologie de l'Hôpital Saint-Louis, vice-présidente de l'Arbre des Connaissances), Christophe Morin (enseignant-chercheur à la faculté des sciences et technologie de l'université Paris-Est Créteil, équipe Glycannes-chimie et biologie dans l'homéostasie tissulaire du laboratoire CRRET ERL-CNRS 9215, responsable de la Cordée de la Réussite Banlieue-Est, cap vers les sciences, président de Promosciences), Marie-José Pestel (présidente du Comité International des Jeux Mathématiques), Sylvie Retailleau (physicienne, présidente de l'université Paris Sud). 


\section{RÉSUMÉS}

L'approche participative est une notion en vogue tant au niveau des politiques publiques qu'à celui des dispositifs de médiation scientifique. Faire intervenir des publics dans la conception d'une exposition scientifique donne une autre dimension à cette participation. Entre théorie de l'action et états des lieux, cette contribution présente QSEC, un dispositif innovant de médiation scientifique.

\section{INDEX}

Mots-clés : claireg@exploradome.com

\section{AUTEURS}

\section{VERA DE SOUSA}

Vera de Sousa a été cheffe de projet à l'association Savoir Apprendre à Vitry-sur-Seine d'octobre 2017 à septembre 2018

\section{CLAIRE GARRAUD}

Claire Garraud est coordinatrice régionale du projet «Questions de Sciences, Enjeux Citoyens » 